\title{
Consumo alimentar conforme pirâmide proposta para pacientes submetidos a cirurgia bariátrica
}

\section{Food consumption according to pyramid proposed for patients undergoing bariatric surgery}

\author{
Fabiana Melo Soares' \\ Epifânio Feitosa da Silva Neto ${ }^{1}$ \\ Caroline Trindade da Silva' \\ Thamires Fernanda Silva Vasconcelos' \\ Oscar Felipe Falcão Raposo² \\ Márcia Ferreira Cândido de Souza ${ }^{3}$ \\ Kiriaque Barra Ferreira Barbosa ${ }^{4}$ \\ ${ }^{1}$ Curso de Nutrição. Universidade Federal de \\ Sergipe. Aracajú, SE, Brasil \\ ${ }^{2}$ Departamento de Estatística e Ciências \\ Atuariais. Universidade Federal de Sergipe. \\ Aracaiú, SE, Brasil \\ ${ }^{3}$ Hospital Universitário da Universidade Federal \\ de Sergipe, Unidade de Alimentação e Nutrição. \\ Aracajú, SE, Brasil \\ ${ }^{4}$ Centro de Ciências Biológicas e da Saúde, \\ Núcleo de Nutrição. Universidade Federal de \\ Sergipe. Aracajú, SE, Brasil \\ Correspondência / Correspondence \\ Kiriaque Barra Ferreira Barbosa \\ E-mail:kiribarra@yahoo.com.br
}

\section{Resumo}

O objetivo deste trabalho foi avaliar a adequação da frequência do consumo alimentar de acordo com pirâmide alimentar para pacientes submetidos a cirurgia bariátrica, com adaptações. Tratou-se de estudo transversal, de amostragem não aleatória por conveniência, no qual foram avaliados 39 pacientes, de ambos os gêneros, faixa etária média de 44,74 anos, submetidos a cirurgia bariátrica e assistidos pelo Sistema Único de Saúde, em nível ambulatorial, no Hospital Universitário de Sergipe. Foram selecionados para participar do estudo os pacientes que apresentavam, no mínimo, dois meses de pós-operatório em cirurgia bariátrica. $\mathrm{O}$ estudo confrontou as porções ingeridas pelos pacientes, obtidas pela média de, pelo menos, dois recordatórios de $24 \mathrm{~h}$ em consultas consecutivas, com as porções recomendadas pela pirâmide alimentar proposta para pacientes em pós-operatório de cirurgia bariátrica. O estado nutricional dos pacientes no pós-operatório está distribuído desde eutrofia até obesidade grau III. O tempo médio de assistência no préoperatório foi de 38,89 meses. Houve inadequação no consumo do grupo das carnes, ovos, leite e derivados e leguminosas; grupo das hortaliças e óleo vegetal e alimentos com alto teor de gorduras e açúcares; adequação no grupo das frutas e grupo dos cereais, raízes e tubérculos. A mudança de hábito alimentar é um processo contínuo e não se refere apenas ao ato cirúrgico. Assim, a construção do conhecimento do padrão alimentar dessa população no longo prazo é de extrema importância, para orientação e prevenção de carências nutricionais.

Palavras-chave: Cirurgia Bariátrica. Período Pós-operatório. Consumo de Alimentos. 


\section{Abstract}

This study aimed to evaluate the adequacy of food consumption frequency according to food pyramid proposed for postoperative patients of bariatric surgery. It is a cross-sectional study, nonrandom sampling by convenience, assessing 39 patients of both genders, aged among 40-59 years, who were submitted to bariatric surgery and assisted by the Brazilian National Health System (SUS), at an outpatient clinic of University Hospital of Sergipe. We selected to participate in the study patients who had at least two months postoperatively in bariatric surgery. The study compared portions taken by patients, based on an average of at least two 24hour dietary recalls on consecutive visits, as recommended by the food pyramid proposed for patients after bariatric surgery. The postoperative nutritional status of patients was distributed from eutrophy to obesity grade III. The average time of preoperative care was 38.89 months. There was inadequacy of consumption level 1 (Group of meat, eggs, dairy products, and legumes); level 2 (Group of vegetables and olive oil) and foods with high fat and sugar; and adequacy at level 2 (fruits group) and level 3 (cereals, roots and tubers). The change in eating habits is a continuous process and does not refer only to the surgical procedure. Thus, the construction of knowledge of the dietary pattern of the population in the long term is paramount for guidance and prevention of nutritional deficiencies.

Key words: Bariatric Surgery. Postoperative Period. Food Consumption.

\section{Introdução}

Para o sucesso no tratamento cirúrgico da obesidade com promoção de perda ponderal satisfatória e manutenção da saúde, é fundamental que o paciente tenha um acompanhamento nutricional direcionado para suas necessidades. Fazem-se imprescindíveis modificações de hábitos alimentares, com auxílio de instrumentos que promovam o entendimento dos pacientes em relação aos grupos alimentares e suas funções, favorecendo a educação nutricional, a fim de promover maior autonomia para adequada seleção dos alimentos, adotando uma dieta equilibrada e variada. ${ }^{1,2}$

Estudos realizados com pacientes em tratamento pós-operatório de cirurgia bariátrica vêm encontrando inadequação alimentar, principalmente em relação aos micronutrientes, o que revela um acompanhamento nutricional insatisfatório e/ou negligência por parte dos pacientes 
em relação à suplementação regular., ${ }^{3,4}$ Segundo Antonini et al., ${ }^{5}$ somente o acompanhamento nutricional adequado promove o sucesso da cirurgia, evitando efeitos colaterais, perda ponderal insuficiente e desnutrição.

Os guias alimentares são instrumentos que visam orientar e informar a população com o intuito de promover hábitos alimentares saudáveis. São caracterizados por diversas representações gráficas da distribuição de alimentos: pirâmides, arco-íris, rodas, entre outros. O conteúdo abordado, assim como a forma gráfica, é frequentemente modificado, conforme novas concepções sobre os alimentos, aspectos culturais, hábitos alimentares e população alvo. ${ }^{1,2,6}$

Diante da necessidade de criar um instrumento para públicos com necessidades específicas como o paciente em tratamento pós-operatório de cirurgia bariátrica, Moizé et al. ${ }^{7}$ propuseram a pirâmide alimentar para esta população, uma vez que possuem especificidades. Trata-se de uma ferramenta construída para apoiar o profissional e os pacientes no aconselhamento, na escolha de alimentos e de suas porções, a fim de proporcionar alimentação e nutrição adequadas no período pós-operatório de uma cirurgia bariátrica. Per si, o mesmo não é capaz de promover perda ponderal. ${ }^{7}$

Diante do exposto e considerando a carência de estudos científicos no Estado de Sergipe, acerca da adequação do consumo alimentar de pacientes submetidos a cirurgia bariátrica, o presente estudo teve como objetivo avaliar a adequação do consumo alimentar atual de acordo com pirâmide alimentar proposta para esta população, a fim de identificar as principais deficiências nutricionais, para orientar e prevenir os distúrbios nutricionais, proporcionando a melhora da qualidade de vida da população em questão.

\section{Materiais e métodos}

Tratou-se de estudo transversal, de amostragem por conveniência composta por 39 pacientes, com idades maiores ou iguais a 18 anos, de ambos os gêneros, submetidos a cirurgia bariátrica e assistidos pelo Sistema Único de Saúde (SUS), em nível ambulatorial, em um hospital universitário de Sergipe. Foram selecionados para participar do estudo todos os pacientes que apresentavam pelo menos dois meses de pós-operatório em cirurgia bariátrica, tendo em vista que a partir deste momento o paciente não possuía alterações acentuadas do padrão alimentar relacionadas a restrições de consistência e volume impostas pela recuperação imediata do procedimento cirúrgico.

Na ocasião das consultas nutricionais, foram coletados dados referentes a identificação pessoal, gênero, idade, tempo de acompanhamento nutricional e de pós-operatório, estilo de vida, hábitos alimentares e estado nutricional do paciente. 
As variáveis peso e estatura foram aferidas seguindo a padronização de Lohman et al.. ${ }^{8}$ A massa corporal (kg) foi mensurada em balança plataforma eletrônica digital (Líder ${ }^{\circledR}$ balanças, modelo LD1050, Araçatuba, São Paulo) com precisão de 50 g, capacidade máxima 200 kg. Para a medição da estatura, foi utilizado o estadiômetro (Tonelli ${ }^{\circledR}$, modelo E150 A, Criciúma, Santa Catarina), com escala em milímetros e amplitude de medida de 400 a 2200 mm, com marcações em milímetros, fixado na parede de superfície plana, sem rodapé e em ângulo de 90 oㅡ com o chão.

Procedeu-se então ao cálculo do índice de massa corporal (IMC). Para a classificação do estado nutricional, foram adotados os pontos de corte preconizados pela Organização Mundial da Saúde. ${ }^{9}$ São Classificados como eutróficos os indivíduos com IMC entre 18,5-24,9 kg/m²; sobrepeso, IMC $\geq 25$. Define-se a obesidade segundo a gravidade em: obesidade grau I quando o IMC situa-se entre 30 e $34,9 \mathrm{~kg} / \mathrm{m}^{2}$, obesidade grau II quando o IMC está entre 35 e $39,9 \mathrm{~kg} / \mathrm{m}^{2}$; e obesidade grau III quando o IMC ultrapassa $40 \mathrm{~kg} / \mathrm{m}^{2} .^{9}$

A pirâmide alimentar proposta por Moizé et al. ${ }^{7}$ para pacientes submetidos a cirurgia bariátrica é composta por cinco níveis. A base da pirâmide corresponde a suplementação, ingestão hídrica e atividade física. Em nosso estudo, utilizamos os níveis referentes apenas ao consumo alimentar. No primeiro nível estão os alimentos fontes de proteína (carnes, ovos, laticínios e leguminosas), com a recomendação diária de 4-6 porções. No segundo nível estão os grupos das frutas, hortaliças e óleos vegetais, com a recomendação diária de 2-3 porções de cada grupo. No terceiro nível estão os cereais, tubérculos e raízes, com a recomendação diária de duas porções. No quarto nível estão as gorduras, doces e bebidas alcoólicas, cujo consumo não é recomendado devido à alta densidade energética.

As adaptações realizadas a partir da pirâmide original consistiram em adotar $50 \mathrm{~g}$ para a porção de pão, equivalente a uma unidade de pão francês, comumente consumido na região. Foram inseridas raízes no grupo dos cereais: macaxeira, inhame e batata doce, integrantes dos hábitos alimentares regionais. O grupo das hortaliças foi subdividido em folhosas e não folhosas; a porção utilizada para as hortaliças folhosas foi de $30 \mathrm{~g}$, correspondendo a três folhas médias de alface. As frutas foram classificadas segundo o teor de carboidratos: fruta A de 5 a $10 \%$, fruta B de 10 a $20 \%$, conforme proposto por Ornellas. ${ }^{10}$

Para avaliar a adequação do consumo alimentar, confrontaram-se as porções ingeridas pelos pacientes, obtidas pela média de, pelo menos, dois a três recordatórios de 24 horas em consultas consecutivas, com as recomendadas pela pirâmide alimentar proposta para pacientes submetidos a cirurgia bariátrica, ${ }^{7}$ adaptada para a realidade local. Considerou-se como adequado o consumo conforme a recomendação. Para o grupo dos alimentos a serem evitados, considerou-se inadequado o consumo desses alimentos, independentemente da quantidade, e adequado para os indivíduos que não consumiram os alimentos, cuja ingestão é desaconselhada (quadro 1). 
Quadro 1. Recomendações das porções dos grupos alimentares para pacientes submetidos a cirurgia bariátrica, Hospital Universitário, Aracaju-SE, 2013.

\begin{tabular}{|c|c|}
\hline Níveis da Pirâmide & 1 Porção $(\mathrm{g})$ \\
\hline \multicolumn{2}{|l|}{ NÍVEL 1- carnes, ovos, leite e derivados e leguminosas: 4 a 6 porções/dia } \\
\hline Ovo & 50 \\
\hline Leguminosas (feijão, lentilha, soja, grão de bico) & 80 \\
\hline Queijo sólido & 50 \\
\hline Queijo pastoso (tipo requeijão) & 80 \\
\hline Leite & 140 \\
\hline Iogurte & 115 \\
\hline Peixe & 85 \\
\hline Carne (bovina, suína ou frango) & 60 \\
\hline \multicolumn{2}{|l|}{ NÍVEL 2 - 2 porções/dia por grupo } \\
\hline Grupo 1: Hortaliças folhosas & 30 \\
\hline Grupo 1: Hortaliças (outros tipos) & 85 \\
\hline Grupo 2: Fruta A & 140 \\
\hline Grupo 2: Fruta B & 70 \\
\hline Grupo 3: Azeite de oliva & 2,4 \\
\hline \multicolumn{2}{|l|}{ NÍVEL 3 - cereais, raízes e tubérculos: 2 porções/dia } \\
\hline Arroz & 90 \\
\hline Massa & 90 \\
\hline Cereais matinais (tipo sucrilhos) & 30 \\
\hline Pão & 50 \\
\hline Torrada & 30 \\
\hline Biscoito & 30 \\
\hline Tubérculos (macaxeira, inhame, batata doce) & 85 \\
\hline \multicolumn{2}{|l|}{ Alimentos que devem ser evitados: ingestão desaconselhada } \\
\hline \multicolumn{2}{|l|}{ Manteiga } \\
\hline \multicolumn{2}{|l|}{ Margarina } \\
\hline \multicolumn{2}{|l|}{ Refrigerantes } \\
\hline Doces & Não ingerir \\
\hline Açúcar de adição & estes \\
\hline Bebida alcoólica & alimentos \\
\hline
\end{tabular}

Adaptado de Moizé et al.(2010). 
A análise estatística descritiva compreendeu o cálculo de média (X), desvio padrão (DP) e frequências absoluta (n) e relativa (\%). Em razão do tamanho amostral e distribuição das variáveis, foram adotados testes não paramétricos. Utilizou-se o teste $X^{2}$ qui-quadrado para comparar a adequação do consumo entre os grupos categorizados pela mediana da distribuição do tempo de tratamento pós-operatório e perda ponderal. Foi considerado o nível de significância estatística de 5\%. As análises estatísticas foram realizadas utilizando o Statistical Package for the Social Science, SPSS versão 20.0 para Windows. ${ }^{11}$

O presente estudo foi aprovado pelo Comitê de Ética em Pesquisa com Seres Humanos da Universidade Federal de Sergipe, Brasil (CAAE - 0153.0.107.000-11), conforme os princípios da declaração de Helsinque.

\section{Resultados}

Dos 39 pacientes submetidos a cirurgia bariátrica, na faixa etária entre 40-59, a maioria foram mulheres. Todos os pacientes, na admissão, mostraram-se com o percentual maior de obesidade grau II ou III e IMC médio de $53,77 \mathrm{~kg} / \mathrm{m}^{2}$, obedecendo aos critérios de indicação ao tratamento cirúrgico da obesidade estabelecido pela Sociedade Brasileira de Cirurgia Bariátrica (tabela 1). ${ }^{12}$

A evolução dos pacientes submetidos ao tratamento cirúrgico da obesidade pode ser evidenciado na melhora do estado nutricional desde a admissão até o pós-operatório tardio, bem como na redução dos valores médios de peso e IMC, mediante assistência nutricional pós-operatória realizada, em média, por 5,37 anos (26,7 meses, segundo a tabela 1 . 
Tabela 1. Caracterização dos pacientes submetidos a cirurgia bariátrica, em assistência ambulatorial, Hospital Universitário, Aracaju-SE, 2012. (n=39)

\begin{tabular}{|c|c|c|}
\hline & $\mathrm{n}$ & $\%$ \\
\hline \multicolumn{3}{|l|}{ Gênero } \\
\hline Feminino & 29 & 74,4 \\
\hline \multicolumn{3}{|l|}{ Faixa etária (anos) } \\
\hline $20-39$ & 13 & 33,3 \\
\hline $40-59$ & 23 & 59,0 \\
\hline$\geq 60$ & 3 & 7,7 \\
\hline \multicolumn{3}{|c|}{ Estado nutricional na admissão } \\
\hline Eutrofia & 0 & 0 \\
\hline Sobrepeso & 0 & 0 \\
\hline Obesidade Grau I & 0 & 0 \\
\hline Obesidade Grau II & 1 & 2,6 \\
\hline Obesidade Grau III & 38 & 97,4 \\
\hline \multicolumn{3}{|c|}{ Estado nutricional na cirurgia ${ }^{a}$} \\
\hline Eutrofia & 0 & 0 \\
\hline Sobrepeso & 0 & 0 \\
\hline Obesidade Grau I & 1 & 2,7 \\
\hline Obesidade Grau II & 6 & 16,2 \\
\hline Obesidade Grau III & 30 & 81,1 \\
\hline \multicolumn{3}{|l|}{ Estado nutricional atual $^{\mathrm{b}}$} \\
\hline Eutrofia & 4 & 10,5 \\
\hline Sobrepeso & 7 & 18,4 \\
\hline Obesidade Grau I & 7 & 18,4 \\
\hline Obesidade Grau II & 8 & 21,1 \\
\hline Obesidade Grau III & 12 & 31,6 \\
\hline
\end{tabular}




\begin{tabular}{lcc}
\hline & $\bar{X}$ & DP \\
\hline Idade & 44,74 & 9.827 \\
Peso Admissão & 140,53 & 31,51 \\
Peso Cirurgia $^{\mathrm{a}}$ & 128,73 & 29,11 \\
Peso Atual b $^{\text {b }}$ & 93,82 & 22,68 \\
IMC Admissão & 53,77 & 11,09 \\
IMC Cirurgia $^{\text {a }}$ & 48,57 & 9,89 \\
IMC Atual $^{\text {b }}$ & 36,18 & 8,88 \\
Tempo de assistência nutricional (anos) & 5,37 & 3,4 \\
Tempo pré-operatório (meses) & 38,89 & 35,44 \\
Tempo pós-operatório (meses) & 26,73 & 16,86 \\
\hline
\end{tabular}

IMC: índice de massa corporal; $n$ : frequência absoluta, \%: frequência relativa, $\bar{X}$ : média; DP: desvio padrão. ${ }^{\mathrm{a}} \mathrm{n}=37 ;{ }^{\mathrm{b}} \mathrm{n}=38$.

${ }^{\text {a, b }}$ perda amostral, dados faltantes nos prontuários dos pacientes.

Como pode ser observado na tabela 2 , a média e a mediana de ingestão dos grupos alimentares estiveram fora da recomendação, exceto para o grupo das frutas e dos cereais. A ingestão de alimentos proteicos esteve aquém da recomendação.

Tabela 2. Consumo alimentar de pacientes submetidos a cirurgia bariátrica, em assistência ambulatorial, Hospital Universitário, Aracaju-SE, 2012. (n=39)

\begin{tabular}{lcccc}
\hline \multicolumn{1}{c}{ Níveis da pirâmide } & $\begin{array}{c}\text { Recomendação } \\
\text { (porção) }\end{array}$ & $\bar{X}$ & DP & Mediana \\
\hline $\begin{array}{l}\text { Nível 1: carnes, ovos, leite e derivados } \\
\text { e leguminosas }\end{array}$ & 4 a 6 & 3,64 & 1,34 & 3,44 \\
Nível 2 & 2 a 3 & 0,96 & 1,08 & 0,71 \\
hortaliças & 2 a 3 & 2,42 & 1,81 & 2,03 \\
frutas & 2 a 3 & 0,21 & 0,66 & 0 \\
azeite de oliva & 2 & 2,17 & 1,29 & 1,97 \\
Nível 3: cereais, raízes e tubérculos & & & & \\
\hline
\end{tabular}

$\overline{\bar{X}}$ : média; DP: Desvio Padrão. 
Na tabela 3, foi possível evidenciar que a inadequação de consumo está direcionada, na sua maioria, para o limite inferior, sendo maior o contingente de pacientes cujo consumo está abaixo da recomendação. Chamam a atenção os grupos das frutas e cereais, raízes e tubérculos, cuja inadequação esteve equilibrada entre os limites inferior e superior da recomendação. Cabe ressaltar que mais de $70 \%$ dos pacientes relataram o consumo de alimentos com alto teor de gordura e açúcar, negligenciando a recomendação de que fossem evitados.

Tabela 3. Adequação do consumo alimentar de pacientes submetidos a cirurgia bariátrica, em assistência ambulatorial, Hospital Universitário, Aracaju-SE, 2012. (n=39)

\begin{tabular}{|c|c|c|c|c|c|c|}
\hline \multirow[t]{2}{*}{ Níveis da pirâmide/ Adequação } & \multicolumn{2}{|c|}{ Abaixo } & \multicolumn{2}{|c|}{ Adequado } & \multicolumn{2}{|c|}{ Acima } \\
\hline & $\mathrm{n}$ & $\%$ & $\mathrm{n}$ & $\%$ & $\mathrm{n}$ & $\%$ \\
\hline $\begin{array}{l}\text { Nível 1: carnes, ovos, leite e derivados e } \\
\text { leguminosas }\end{array}$ & 28 & 71,8 & 8 & 20,5 & 3 & 7,7 \\
\hline \multicolumn{7}{|l|}{ Nível 2} \\
\hline hortaliças & 33 & 84,6 & 4 & 10,3 & 2 & 5,1 \\
\hline frutas & 19 & 48,7 & 7 & 18,2 & 13 & 33,3 \\
\hline azeite de oliva & 37 & 94,9 & 2 & 5,1 & 0 & 0 \\
\hline \multirow[t]{2}{*}{ Nível 3: cereais, raízes e tubérculos } & 20 & 51,3 & 0 & 0 & 19 & 48,7 \\
\hline & \multicolumn{3}{|c|}{ Adequado } & \multicolumn{3}{|c|}{ Inadequado } \\
\hline \multirow{2}{*}{$\begin{array}{l}\text { Nível 4: alimentos com alto teor } \\
\text { de gorduras e açucares (doces, } \\
\text { refrigerantes, frituras, bebidas alcoólicas) }\end{array}$} & $\mathrm{N}$ & & $\%$ & $\mathrm{~N}$ & & $\%$ \\
\hline & 11 & & 28,2 & 28 & & 71,8 \\
\hline
\end{tabular}

n: frequência absoluta, \%: frequência relativa.

Quando comparada a adequação do consumo dos grupos alimentares dos pacientes em relação à mediana do tempo de cirurgia, houve significância estatística apenas no nível 2, grupo das frutas, sendo observado que a adesão ao consumo recomendado de frutas foi maior entre aqueles pacientes com menor tempo de acompanhamento nutricional no pós-operatório (tabela 4). 
Tabela 4. Adequação do consumo dos grupos alimentares, de pacientes submetidos a cirurgia bariátrica em assistência ambulatorial, segundo mediana do tempo de tratamento pós-operatório, Hospital Universitário, Aracaju-SE 2012. (n=39)

\begin{tabular}{lcccccc}
\hline & & \multicolumn{2}{c}{$<27$ meses } & \multicolumn{2}{c}{$\geq 27$ meses } & P \\
& & $\mathrm{n}^{1}$ & $\%$ & $\mathrm{n}^{2}$ & $\%$ & \\
\hline Nível 1: Carnes, Ovos, Leite e & Abaixo & 13 & 72,2 & 15 & 71,4 & 0,884 \\
Derivados e Leguminosas & Adequado & 4 & 22,2 & 4 & 19 & \\
& Acima & 1 & 5,6 & 2 & 9,5 &
\end{tabular}

Nível 2

Hortaliças

$\begin{array}{ccccc}\text { Abaixo } & 17 & 94,4 & 16 & 76,2 \\ \text { Adequado } & 1 & 5,6 & 3 & 14,2 \\ \text { Acima } & 0 & 0 & 2 & 9,5\end{array}$

Frutas

$\begin{array}{ccccc}\text { Abaixo } & 5 & 27,8 & 14 & 66,7 \\ \text { Adequado } & 5 & 27,8 & 2 & 28,6 \\ \text { Acima } & 8 & 44,4 & 5 & 23,8\end{array}$

Azeite de Oliva

$\begin{array}{cccccc}\text { Abaixo } & 17 & 94,4 & 20 & 95,2 & 0,911 \\ \text { Adequado } & 0 & 0 & 0 & 0 & \\ \text { Acima } & 1 & 5,6 & 1 & 4,8 & \end{array}$

Nível 3: Cereais Raízes e Tubérculos

$\begin{array}{ccccc}\text { Abaixo } & 9 & 50 & 11 & 52,4 \\ \text { Adequado } & 0 & 0 & 0 & 0 \\ \text { Acima } & 9 & 50 & 10 & 47,6\end{array}$

Nível 4: Alimentos com alto teor

$\begin{array}{ccccc}\text { Adequado } & 4 & 22,2 & 7 & 33,3 \\ \text { Inadequado } & 14 & 77,8 & 14 & 66,7\end{array}$

de gorduras e açucares (doces,

$\begin{array}{lllll}\text { Inadequado } & 14 & 77,8 & 14 & 66,7\end{array}$ refrigerantes, frituras)

n: frequência absoluta, \%: frequência relativa. ${ }^{*} \mathrm{p}<0,05$, Teste Qui-Quadrado, ${ }^{1} \mathrm{n}=18 ;{ }^{2} \mathrm{n}=21$

A ingestão foi considerada adequada quando atingiu a recomendação da pirâmide alimentar proposta para pacientes em tratamento pós operatório de cirurgia bariátrica 
Quando comparada a adequação do consumo dos grupos alimentares em relação à mediana da perda ponderal, não houve significância estatística para nenhum dos grupos da pirâmide alimentar (tabela 5).

Tabela 5. Adequação do consumo dos grupos alimentares de pacientes em tratamento pósoperatório de cirurgia bariátrica em assistência ambulatorial, segundo mediana de perda ponderal no pós-operatório. Aracaju-SE, 2012. (n=37)

\begin{tabular}{|c|c|c|c|c|c|c|}
\hline & & \multicolumn{2}{|c|}{$<22 \mathrm{Kg}$} & \multicolumn{2}{|c|}{$\geq 22 \mathrm{~kg}$} & \multirow[t]{2}{*}{$\mathrm{P}$} \\
\hline & & $\mathrm{n}^{1}$ & $\%$ & $\mathrm{n}^{2}$ & $\%$ & \\
\hline Nível 1: Carnes, Ovos, Leite e & Abaixo & 14 & 77,8 & 13 & 73 & 0,366 \\
\hline \multirow[t]{2}{*}{ Derivados e Leguminosas } & Adequado & 4 & 22,2 & 4 & 21,6 & \\
\hline & Acima & 0 & 0,0 & 2 & 5,4 & \\
\hline \multicolumn{7}{|l|}{ Nível 2} \\
\hline \multirow[t]{3}{*}{ Hortaliças } & Abaixo & 17 & 94,4 & 14 & 73,7 & 0,195 \\
\hline & Adequado & 4 & 5,6 & 3 & 15,8 & \\
\hline & Acima & 1 & 0 & 2 & 10,5 & \\
\hline \multirow[t]{3}{*}{ Frutas } & Abaixo & 5 & 27,8 & 12 & 63,2 & 0,089 \\
\hline & Adequado & 5 & 27,8 & 2 & 10,5 & \\
\hline & Acima & 8 & 44,4 & 5 & 26,3 & \\
\hline \multirow[t]{3}{*}{ Azeite de Oliva } & Abaixo & 17 & 94,4 & 18 & 94,7 & 0,969 \\
\hline & Adequado & 1 & 5,6 & 1 & 5,3 & \\
\hline & Acima & 0 & 0 & 0 & 0 & \\
\hline \multirow{4}{*}{$\begin{array}{l}\text { Nível 3: Cereais, Raízes e } \\
\text { Tubérculos }\end{array}$} & Abaixo & 11 & 61,1 & 8 & 42,1 & 0,248 \\
\hline & & & & & & \\
\hline & Adequado & 0 & 0 & 0 & 0 & \\
\hline & Acima & 7 & 38,9 & 11 & 57,9 & \\
\hline \multirow{2}{*}{$\begin{array}{l}\text { Nível 4: Alimentos com alto } \\
\text { teor de gorduras e açucares } \\
\text { (doces, refrigerantes, frituras) }\end{array}$} & Adequado & 6 & 38,9 & 5 & 26,3 & 0,641 \\
\hline & Inadequado & 12 & 61,1 & 14 & 73,7 & \\
\hline
\end{tabular}

n: frequência absoluta, \%: frequência relativa.

Teste Qui-Quadrado, ${ }^{1} \mathrm{n}=18 ;{ }^{2} \mathrm{n}=19$

* Durante a coleta de dados anteriores houve perda de dados referentes a antropometria de dois pacientes A ingestão foi considerada adequada quando atingiu a recomendação da pirâmide alimentar proposta para pacientes em tratamento pós operatório de cirurgia bariátrica 


\section{Discussão}

A população do presente estudo foi predominantemente feminina, corroborando os estudos de Oliveira et al., ${ }^{13}$ Santos, Burgos \& Silva ${ }^{14}$ e Quadros et al., ${ }^{15}$ cujos achados foram 84,4\%, 75\% e $77,5 \%$, respectivamente.

As mulheres sofrem maior pressão em relação aos padrões estéticos valorizados pela sociedade, podendo haver relação com a maior motivação para o tratamento cirúrgico da obesidade. ${ }^{16-18} \mathrm{~A}$ população foi composta de indivíduos adultos, com média de idade 44,74 anos, faixa etária dentro do critério idade para submissão do tratamento cirúrgico da obesidade grave (18-65 anos).12,19

A inadequação do consumo de alimentos (fontes proteicas de alto valor biológico) relacionados ao nível 1 da pirâmide alimentar proposta para paciente em tratamento pós operatório, esteve elevada tanto para os pacientes recém-operados como para aqueles em tratamento pós-operatório tardio. A deficiência proteica promove sintomas característicos, a saber: hipoalbuminemia, redução de massa magra, anemia, edema, astenia e alopecia. A redução da ingestão tem como principal fator a restrição mecânica, o tamanho reduzido do estômago e a disponibilidade reduzida de pepsina e ácido clorídrico. ${ }^{20-22}$

Essa população recebeu, continuamente, orientação sobre a necessidade do consumo de alimentos fonte de proteína e a importância da suplementação regular. Ainda assim, apenas uma pequena parcela dos pacientes aderiu a sua utilização regular. Estudo prévio de Silva et al., ${ }^{23}$ realizado com a mesma população do presente estudo, revelou que a aceitação da suplementação foi ruim entre $50 \%$ dos pacientes que faziam uso regular do suplemento e entre $26,1 \%$ daqueles pacientes que negligenciavam a recomendação da utilização regular do suplemento proteico. Desses, 80\% atribuíram a não aceitação ao sabor/odor desagradável característicos do produto, e $20 \%$ a sintomas relacionados ao trato gastrointestinal. Bordalo et al. ${ }^{24}$ ratificam que alguns fatores interferem na aceitação da suplementação proteica, como, sabor, textura, solubilização, absorção e custo.

Diante da não aceitação dos suplementos proteicos, além do relato de seus efeitos adversos, uma conduta adotada no serviço era diminuir ou isentar os pacientes da sua utilização regular. No entanto, esta conduta era restrita aos pacientes que já estavam em pós-operatório tardio que conseguiam manter uma ingestão proteica adequada e cujos exames bioquímicos respaldavam que as dosagens sanguíneas de proteínas e frações se mantinham dentro do padrão de referência.

No longo prazo, o aumento do consumo de energia pode estar relacionado com a melhor tolerância alimentar, bem como maior adaptação gástrica, melhorando a eficiência da absorção de proteína. A suplementação é indicada quando não há tolerância dos alimentos-fontes, completando uma dieta mista. ${ }^{25,26}$ 
A ingestão inadequada deste grupo pode ser influenciada por situações que cercam a cirurgia bariátrica, incluindo intolerâncias alimentares, anorexia, vômitos, diarreia, medo de reganho de peso, depressão, álcool, nível socioeconômico, dietas restritas aliadas a modificações na percepção do sabor e escolhas alimentares que contribuem para a deficiência, não só proteica, mas também de micronutrientes. ${ }^{20-22}$ Segundo Westerterp-Plantenga et al., ${ }^{27}$ a ingestão elevada de proteínas está relacionada a melhor evolução ponderal e preservação de massa magra.

A ingestão adequada do grupo das frutas repercute no incentivo ao consumo de vegetais de baixa densidade calórica, rico em fibras, minerais e vitaminas, favorecendo a plenitude e a saciedade. ${ }^{28} \mathrm{O}$ consumo adequado no grupo das frutas pode estar relacionado com o fácil acesso, disponibilidade local e baixo preço relativo. A menor adesão ao consumo dos que apresentavam maior tempo de acompanhamento, se deveu possivelmente ao fato de esses pacientes, com maior tempo de acompanhamento pós-operatório, serem aqueles que perderam a adesão ao tratamento, deixando de ir às consultas e/ou se tornaram mais permissivos, negligenciando as orientações. ${ }^{1,29}$

Quanto maior for o tempo posterior à realização da cirurgia, mais esparsas se tornam as frequências das consultas de assistência nutricional pós-operatória. Assim, diante da remissão dos sintomas, os pacientes tendem a se tornar mais independentes e confiantes, o que por sua vez os torna mais permissivos, resultando na prática de hábitos alimentares inadequados. Esta maior permissividade em relação às escolhas alimentares tem influência no reganho de peso.,29-31

O elevado contingente de pacientes $(71,8 \%)$ que relataram consumir alimentos com alto teor de açúcar e gordura pode ser devido ao fato de estes serem palatáveis, de fácil ingestão e exigirem menor esforço na mastigação e/ou denota uma negligência importante quanto às orientações de limitar seu consumo, visto que tais alimentos não favorecem a saciedade e comprometem a perda ponderal. O consumo desses alimentos foi observado tanto em pacientes no pós-imediato como no pós-tardio. Esses alimentos podem limitar a perda de peso, além de desencadear efeitos deletérios, como síndrome de dumping e intolerância alimentar. ${ }^{15,32}$

\section{Conclusão}

O consumo alimentar dos pacientes submetidos a cirurgia bariátrica, mediante a pirâmide alimentar proposta para este grupo, mostrou-se adequado em relação aos grupos das frutas e dos cereais, raízes e tubérculos, e inadequado para os grupos das carnes, ovos, laticínios e leguminosas e das hortaliças e óleos vegetais, o que evidencia possíveis riscos nutricionais. A mudança de hábito alimentar é um processo continuo; os esclarecimentos sobre alimentação e nutrição devem preceder a cirurgia e ser construídos de modo a alicerçar uma educação alimentar duradoura.

Assim, é de extrema importância a construção do conhecimento do consumo alimentar dessa população, com vistas a orientação e prevenção de carências nutricionais. 


\section{Referências}

1. Faria SL, et al. Sugestão do uso da pirâmide alimentar em cirurgia bariátrica. Nutr. Pauta. 2008;92(16):14-20.

2. Philippi ST, et al. Pirâmide alimentar adaptada: guia para escolha dos alimentos. Rev Nutr. 1999;12(1):65-80.

3. Harbottle L. Audit of nutritional and dietary outcomes of bariatric surgery patients.Obesity reviews. 2010;12:198-204.

4. Prevedello CF, et al. Análise quantitativa e qualitativa da dieta de pacientes no pré e pós-operatório de cirurgia bariátrica. Bras Nutr Clin. 2009;4(3):159-65.

5. Antonini DR, et al. Avaliação nutricional dos pacientes submetidos à cirurgia bariátrica. Bol Cirur Obes. 2001;2(4):3.

6. Welsh S, Davis C, Shaw A. Development of the food guide pyramid. Nutrition Today. 1992;27(6):12-23.

7. Moizé VL, et al. Nutritional pyramid for post-gastric bypass patients. Obes Surg. 2010;20:1133-41.

8. Lohman TG, Roche AF, Martorell R. Anthropometric Standardization Reference Manual. Champaign, Illinois: Human Kinetics; 1988.

9. World Health Organization. Obesity: preventing and managing the global epidemic. Report of a World Health Organization Consultation. Geneve: WHO; 2000.

10. Ornellas LH. Técnica Dietética, Seleção e Preparo de alimentos. 8. ed. São Paulo: Atheneu; 2007.

11. Statistical package for the social science for windows. SPSS/Pc version 20.0, Chicago: SPSS; 2011.

12. Sociedade brasileira de cirurgia bariátrica e metabólica. Consenso Brasileiro Multissocietário em Cirurgia da Obesidade. São Paulo: 2006. Disponível em: http://www.sbcb.org.br/associados. asp?menu=2

13. APF, et al. Perfil de pacientes submetidos à cirurgia bariátrica atendidos em um hospital universitário do município de São Paulo. Saúde Coletiva. 2009;6(35):275-9.

14. Santos MC, Burgos MGPA, Silva SA. Perda ponderal após cirurgia bariátrica de Fobi-Capella: realidade de um hospital universitário do nordeste brasileiro. Rev Bras Nutr Clin. 2006;21(3):188-92.

15. Quadros MRR, et al. Intolerância alimentar no pós-operatório de pacientes submetidos à cirurgia bariátrica. Rev Bras Nutr Clin. 2007;22(1):15-9.

16. Fiates GMR, Salles RK. Fatores de risco para o desenvolvimento de distúrbios alimentares: um estudo em universitárias. Rev Nutrição. 2001;14(Suppl 0):3-6.

17. Quadros MRR, Branco AJ, Zacarias JA. Análise da evolução dietética no pós-operatório de cirurgia bariátrica. Nutr Pauta. 2005 Mai/Jun;13-8.

18. Mazzeo SE, Saunders R, Mitchell KS. Binge eating among African American and Caucasian bariatric surgery candidates. Eat Behav. 2005;6:189-96. 
19. NIH Consensus Statement, Gastroitestinal Surgery for Severe Obesity Nutrition the International Journal of Applied and Basic Nutritional; 1996.

20. Bloomber RD, et al. Nutritional deficiencies following bariatric surgery: what have we learned? Obes Surg. 2005;15:145-54.

21. Burgos GPA, Salviano FN. Complicações nutricionais no Pós-Operatório: Precoces e Tardias. In: Burgos GPA, Lima DSC, Coelho PBP. Nutrição em Cirurgia Bariátrica. Rio de Janeiro: Editora Rubio; 2011. p.131-50.

22. Soares CC, Falcão MC. Abordagem nutricional nos diferentes tipos de cirurgia bariátrica. Rev Bras Nutr Clin. 2007;22(1):59-6.

23. Silva CT, Vasconcelos TFS, Soares FM, Neto EFS, Barbosa KBF. Avaliação da ingestão protéica e uso de suplementos em pacientes em tratamento pós-operatório de cirurgia bariátrica, assistidos em nível ambulatorial. In: $22^{\circ}$ Encontro de Iniciação Científica; 2012; São Cristóvão.

24. Bordalo LA, et al. Cirurgia bariátrica: como e por que suplementar. Rev Assoc Med Bras. 2011;57(1):113-20.

25. Aillsl, et al. ASMBS Allied Health Nutritional Guidelines for the Surgical Weight Loss Patient. Surg Obes Relat Dis. 2008;4(5):S73-108.

26. Pories WJ, et al. Who would have thought it? An operation proves to be the most effective therapy for adult-onset diabetes mellitus. Ann Surg. 1995;222(3):339-52.

27. Westerterp-Plantenga MS, et al. Dietary protein, weight loss, and weight maintenance. Annu Rev Nutr. 2009.29:21-41.

28. Sarwer DB, et al. Dietary intake and eating behavior after bariatric surgery: threats to weight loss maintenance and strategies for success. b. Surg Obes Relat Dis. 2011;7:644-51.

29. Cambi MPC, Marchesini JB. Acompanhamento clínico, dieta e medicação. In: Garrido Jr AB, editor. Cirurgia da obesidade. São Paulo: Atheneu; 2003.

30. Suter M, et al. A New Questionnaire for Quick Assessment of Food Tolerance after Bariatric Surgery. Obes Surg. 2007;17:2-8.

31. Silva MRSB, Silva SRB, Ferreira AD. Intolerância alimentar pós-operatória e perda de peso em pacientes submetidos à cirurgia bariátrica pela técnica Bypass Gástrico. J Health Sci Inst. 2011;29(1):p.41-4.

32. Swenson BR, et al. The effect of a lowcarbohydrate, high-protein diet on post laparoscopic gastric bypass weight loss: a prospective randomized trial. J Surg Res. 2007;142(2):308-13.

Recebido: 11/6/2013

Revisado: 05/8/2013

Aprovado: 06/7/2013 
